\title{
ReVIEW | Legions of Boom: Filipino American Mobile DJ Crews in the San Francisco Bay Area
}

\author{
Oliver Wang
}

Durham, US: Duke University Press, 2015

ISBN: 978-0-8223-5904-3 (HB) / 978-0-8223-5890-9 (PB)

\author{
Brian Fauteux \\ University of Alberta, Canada \\ fauteux@ualberta.ca
}

Throughout American popular music history, local music scenes have developed distinct sounds, styles, dances, and performances. These scenes create communities and bring people together to share in the collective experience of music. What these scenes do not all share in common, however, is the degree to which each has been recorded and written into popular history. In Legions of Boom, Oliver Wang details the history and inner workings behind one particular scene that has yet to be the focus of a major scholarly work: the Filipino American mobile DJ crews of the San Francisco Bay Area. These crews, active in the late 1970s and into the mid-1990s, would influence the DJ cultures and practices of West Coast hip-hop and dance music that followed in their wake.

As the book effectively explains, one major reason as to why the history of Filipino American mobile DJ crews is not widely known is because the crews focused primarily on performance, much more so than recording or documentation. As Wang notes, mobile crews shared the core mission of keeping people dancing, "whether through their mixing techniques, song selections, lighting displays, or enthused exhortations from the DJs themselves, urging crowds to get on up, then get on down" (9). Unlike other DJ-centric scenes - hip-hop, house, reggae, and so forth - the mobile crew DJs did not make the jump from 
"record-playing to record-making" (10). Also, an absence of mass media coverage of the scene contributes to the little attention it has received.

The scene was a dominant part of the recreational and social lives of Filipino American youth in the Bay Area. Participants included DJs, the support teams that would help transport and set up the sound and lighting systems, and the individuals who attended the weddings, school dances, and parties that the mobile crews performed at. Wang traces the birth of the scene from spaces such as garages and school gymnasiums to the larger DJ showcases and battles that would pit mobile crews against one another. He pairs numerous interviews with scene participants with that of popular music studies and cultural studies literature in order to make sense of the ways in which the mobile DJ crew scene was imbued with issues of gender, community, and capital. Drawing heavily on the concepts of cultural and symbolic capital, Wang communicates the importance of social capital to the mobile DJ crews. Surrounding the scene was a network of friends, family, and community members who helped sustain paying gigs for different crews.

The reader is first introduced to the preconditions of the mobile scene, emphasizing the coming together of aesthetic innovations in DJ culture, the immigration and settlement patterns of Filipino American families, and the tradition of garage parties. The garage is a particularly significant space within this scene, as it offered youth a place to gather and experiment with technologies and techniques, as well as a social space to host dance parties. The discussion then moves from the garage to the school dance, where many mobile crews got their start. In the discussion that follows, community and creative copyright are the focus, as mobile crews participate in showcases and battles, competing for symbolic capital and working to build reputations. These showcases also served to maximize audience participation and to include venues all around the Bay Area.

Wang argues that the scene declined when individual scratch DJs became the focus of showcases. To chart this transition, Wang likens the DJ to a drummer: "the best mixers sustain a danceable backbeat, but the scratch DJ is all about the drum solo" (130). Scratch DJs do not require a support team, or a crew, for a performance. Sets are shorter and the focus is on the individual. The transition between mobile crews to individual scratch DJs also anticipates a larger shift to digital technology in the popular music industry. One in which portability is key. Although this is not a focus of the book, the mobile scene can indeed be considered in this larger industry context as well.

A major strength of Legions of Boom is the thorough and careful definitions offered by Wang each time a new term or concept, specific to the mobile DJ crews, is introduced. Some of these terms include "nonstop disco mixing", "slipcueing", and "disco break". For readers unfamiliar with the scene that Wang chronicles, this is a helpful aspect of the work. The book is also clearly written and is accessible to a wide variety of readers.

Legions of Boom makes a fascinating contribution to popular music history and to studies of music scenes by sharing the practices behind the mobile DJ crews of the Bay Area. Wang concludes by noting that his work scrapes "the proverbial surface" and that he has "asked a select and relatively small set of questions for a community, scene, and movement that is ripe for far greater exploration and 
examination" (158). By studying musical moments that have managed to slip by processes of documentation, we can share the stories and experiences of groups and individuals who sustain local scenes, and help to broaden our understanding of popular music history. 